\title{
The MicroAce: An inexpensive computer controller
}

\author{
JERRY W. O'DELL \\ Eastern Michigan University, Ypsilanti, Michigan 48197
}

\begin{abstract}
A 8200 computer system is described that can act as a laboratory controller. Advantages and disadvantages of the system are discussed.
\end{abstract}

Many small computers are available for use in the psychology laboratory (e.g., the Apple, TRS-80, and the Kim). These are well summarized by Reed (1980). The really inexpensive machines, such as the Kim, must be programmed exclusively in machine language, and the more sophisticated machines, with BASIC, cost nearly $\$ 1,000$.

This paper describes the MicroAce system, which can be obtained in kit form, with $2 \mathrm{~K}$ memory, for $\$ 149$. A prewired virtually identical machine is the Sinclair ZX80 , priced at $\$ 200$. A television-set monitor is not included. The computer can be constructed from the kit in about $3 \mathrm{~h}$.

The MicroAce is a fully functional and usable computer with a built-in video generator and a very decent integer BASIC. The basic microprocessor is the Z80A, by Zilog. The unit also has PEEK, POKE, and USR functions, which make it possible to use machine language directly. This is essential if the user wishes to use the machine as a controller for experiments. The use of the Z80A in the MicroAce means that it is possible to use the excellent series of support chips made by Zilog. These devices, once their operation is understood, make interfacing the Z80A to the outside world very simple.

\section{A DEMONSTRATION EXPERIMENT}

As an illustration of the potential power of the MicroAce, assume the laboratory has a single operant chamber, with two cue lights, a single lever, and a pellet dispenser. An animal placed in the box is confronted with a 5 -sec VI schedule associated with Cue Light 1, and a 10-sec VI schedule with Cue Light 2. Ten intervals are used in each condition, and they alternäte. The animal is reinforced only after responding at least once in each time period. The computer generates the timing intervals, controls the distribution of reinforcements, and counts the leverpresses under the Cue-Light 1 and Cue-Light 2 conditions.

\section{PROCEDURE}

In addition to the MicroAce computer, the interfacing equipment detailed in Figure 1 is required. A Scientific Prototype A105 operant chamber, with a $+24-\mathrm{V}$ power source is cited in the schematic diagram.

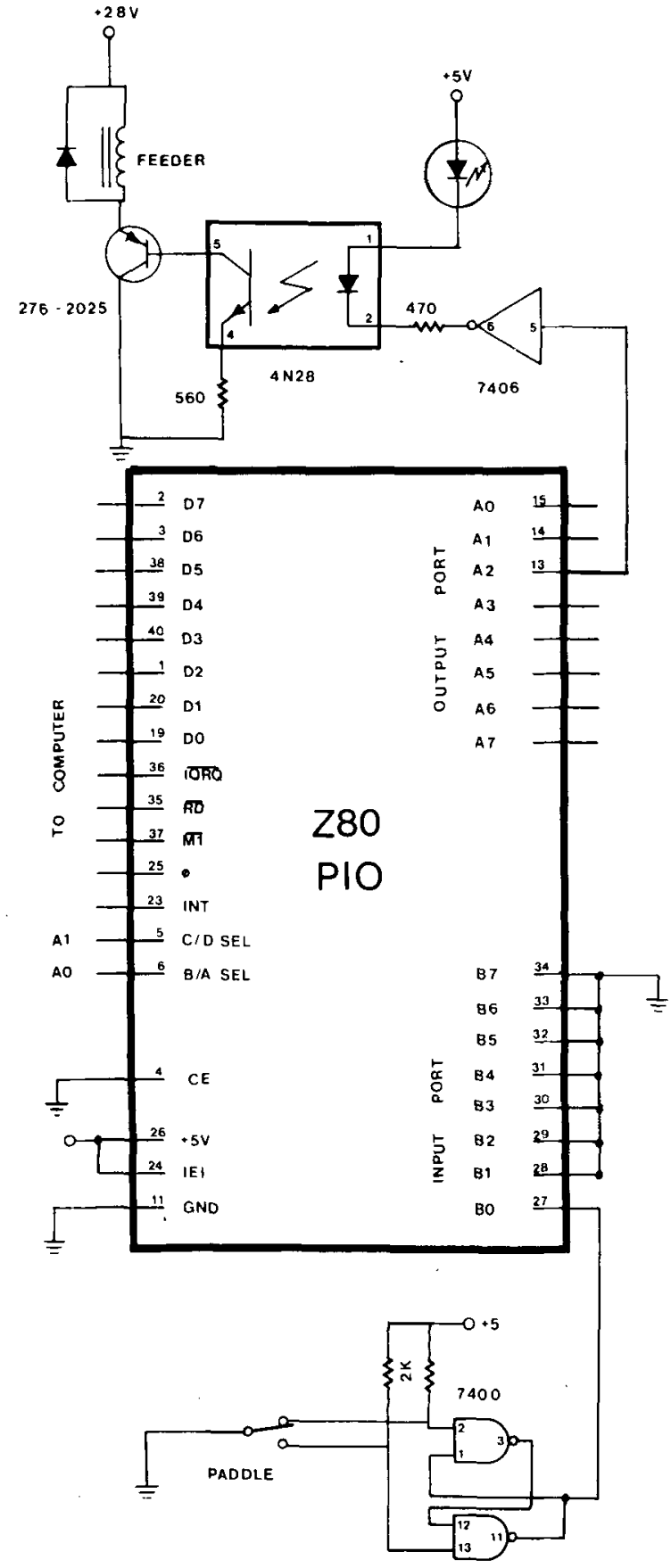

Figure 1. Circuit diagram. 
The heart of the unit is the Zilog Z80A-PIO chip (parallel input/output) which saves endless grief in interfacing and costs $\$ 10$. The appropriate pins of the PIO are hooked up to the same pins on the MicroAce, and the major part of the interface is completed.

The PIO can be used in many modes. A full description of the unit and its operation can be found in Nichols, Nichols, and Rony (1979). In the present application, the PIO Port A is used as output and Port B as input.

The pins in the upper left-hand corner of the PIO chip in the diagram constitute the interface between the MicroAce and the PIO. Functions D7 through INT are interconnected directly. C/D SELECT is connected to address line A1, and B/A SELECT is connected to A0. This makes the input/output addresses of the PIO as follows: A-DATA, 00; B-DATA, 01; A-CONTROL, 02; B-CONTROL, 03. This simple scheme works well. The connections between the PIO and the computer should be as short as possible and no longer than $20 \mathrm{~cm}$. MicroAce outputs are not buffered.

\section{INPUT CIRCUITRY}

The input circuitry is shown at the bottom of Figure 1. A 7400 integrated circuit is used in conjunction with the SPDT switch on the paddle to make a "bounceless" pushbutton. The circuit shown eliminates the contact bounce problem. Further signal conditioning can be provided by a 74121 one-shot. The output of the 7400 is connected to lead B0 of the PIO (Pin 27). It is important to ground the unused input pins, as shown.

\section{OUTPUT CIRCUITRY}

Three output connections are needed, one for each cue light and one for the feeder mechanism. Light 1 is connected to lead A0 of the PIO, Light 2 is connected to lead A1, and the feeder mechanism to A2. The feeder is by far the most difficult device to interface, and consequently it is shown on the diagram. Actually, the three interfaces are almost identical.

The signal to the feeder (Pin 13 of the PIO) is routed through an opto-isolator, the 4 N28. A 7406 integrated circuit drives the opto-isolator; another light-emitting diode is in series with both to provide visual confirmation of the circuit's operation. The $4 \mathrm{~N} 28$ completely separates the computer system, electrically, from the feeder mechanism. It also simplifies the interfacing of modern TTL circuitry with the older $+24-\mathrm{V}$ circuitry used in psychological laboratories.

The feeder is driven by a single transistor, in this case a Radio Shack 276-2025. Any fairly high-powered PNP transistor will generally suffice. Because of the large amount of current (1A), it is essential that the transistor be provided with a heat sink of sufficient size-a sheet of aluminum is sufficient. The collector of the transistor is grounded and can be mounted directly to the mounting enclosure, if desired. The inductive kick of the feeder coil is handled by the diode connected across the coil. Any small silicon diode with ratings of $1 \mathrm{~A}$ and 400 PIV can be used. The diode effectively shorts out the inductive kick of the coil. Without it, the transistor may be destroyed.

The interfaces for the lights are almost identical with that for the feeder. In the case of the lights, however, a heat sink is not necessary.

\section{PROGRAM}

The computer software that controls the experiment is listed in Figure 2. Lines 10-190 contain the machine language that controls the input and output functions. Lines 300-440 take the machine code from the "remark" lines, convert it into the proper values, and place it in memory starting at Location 18001 . The process stops when a sign for "pound sterling" is encountered.

Lines 490-790 are the BASIC program that controls the experiment.

The program begins at line 490 by setting up variable $\mathrm{E}$, the number of passes through the main loop, at 10 . In line 500 , a call is made to a machine language subroutine (at 18001) which sets up Port A of the PIO as output, Port $B$ as input, turns off all the output lines, and clears Memory Location 18200, in which the current status of the lights and feeder is kept. In line 510 , a call is made to Machine Location 18097 (originally on remark line 130), which turns on Cue Light 1 . Then, in lines 520 to 530 , the two counters for the two conditions are set equal to zero.

The main loop in the program uses index $\mathrm{J}$, and goes from line 540 to line 770 . In line 550 , the time for the first portion of the variable-interval schedule associated with Cue Light 1 is stored in Location 18035; the actual values for the times, in seconds, are obtained beginning at 18121 and 18137 (remark lines 160 to 190).

In line 560 , a call is made to the machine language routine starting at Location 18017 (remark lines 30 to 120 ). This is a somewhat tricky routine that does the timing in seconds and at the same time scans the input lines B0 through B7, making a tally in Locations 18220 to 18227 when a key is pressed. Each line is scanned on the order of 3,000 times/sec.

The timing of the interval is set by the constants in the machine language program found at Locations 18037 and 18039. In the present case, these are 40 and $3 F$ (hexadecimal), respectively. With the crystal oscillator in the writer's MicroAce, these values give precisely $1 \mathrm{sec}$ of timing for each count in Memory Location 18035; other users might have to vary these values to suit their crystals. All of these constants are found in remark line 50 in the program. 
10 REM 3E $9 \mathrm{~F}$ D3 92 3E $4 \mathrm{~F}$ D3 $\emptyset 3$

20 REM 3E $\emptyset \emptyset \quad 32 \quad 18 \quad 47 \quad$ D3 $\emptyset \emptyset \quad$ C9

30 REM DD 21 2C $47 \quad \emptyset 6 \quad 9897 \quad$ DD

40 REM $77 \emptyset \emptyset$ DD 23 1ø F9 D9 4F

50 REM D9 IE 10 16 40 वE $3 F$ DD

$\begin{array}{lllllllll}60 & \text { REM } 21 & 2 C & 47 & 96 & 98 & \text { DB } & 91 & \text { D9 }\end{array}$

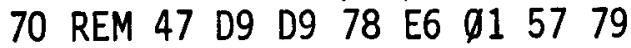

80 REM E6 91 5F BA 28 ดC CB 47

90 REM $28 \quad 93$ DD $34 \quad 90 \quad 79$ CB 87

100 REM 82 4F CB 99 CB 98 DD 23

110 REM D9 $1 \varnothing$ DF $\emptyset \mathrm{D} 2 \emptyset$ D1 $152 \emptyset$

120 REM CC $102 \emptyset$ C7 C9 $\emptyset \emptyset \quad \emptyset \emptyset \emptyset \emptyset$

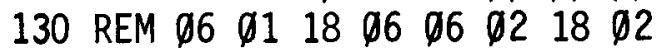

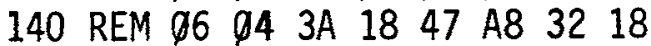

150 REM 47 D3 $\emptyset \emptyset \quad C 9 \quad \emptyset \emptyset \emptyset \emptyset \emptyset \emptyset \emptyset \emptyset$

$\begin{array}{llllllllll}160 & \text { REM } & 91 & 99 & 92 & 98 & 93 & 97 & 94 & 96\end{array}$

170 REM $\emptyset 2 \quad \emptyset 8 \quad \emptyset \emptyset \emptyset \emptyset \emptyset \emptyset \quad \emptyset \emptyset \emptyset \emptyset \emptyset \emptyset$

$\begin{array}{llllllll}180 & \text { REM } \emptyset 4 & 12 & \emptyset \mathrm{E} & \emptyset 6 & \emptyset 2 & 1 \emptyset \emptyset \mathrm{C} & \emptyset 8\end{array}$

190 REM $\emptyset 41 \emptyset £$

300 LET $K=16423$

310 LET $L=1809 \emptyset$

320 LET $K=K+4$

330 FOR $I=1$ TO 8

340 LET $A=\operatorname{PEEK}(K)$

350 LET $B=\operatorname{PEEK}(K+1)$

360 IF $A=12$ THEN GO TO 440

370 LET $C=(A-28) * 16+B-28$

380 LET $L=L+1$

390 POKE L,C

400 LET $K=K+3$

410 IF I $=8$ THEN LET $K=K-1$

420 NEXT I

430 GO TO 320

440 PRINT "DONE"

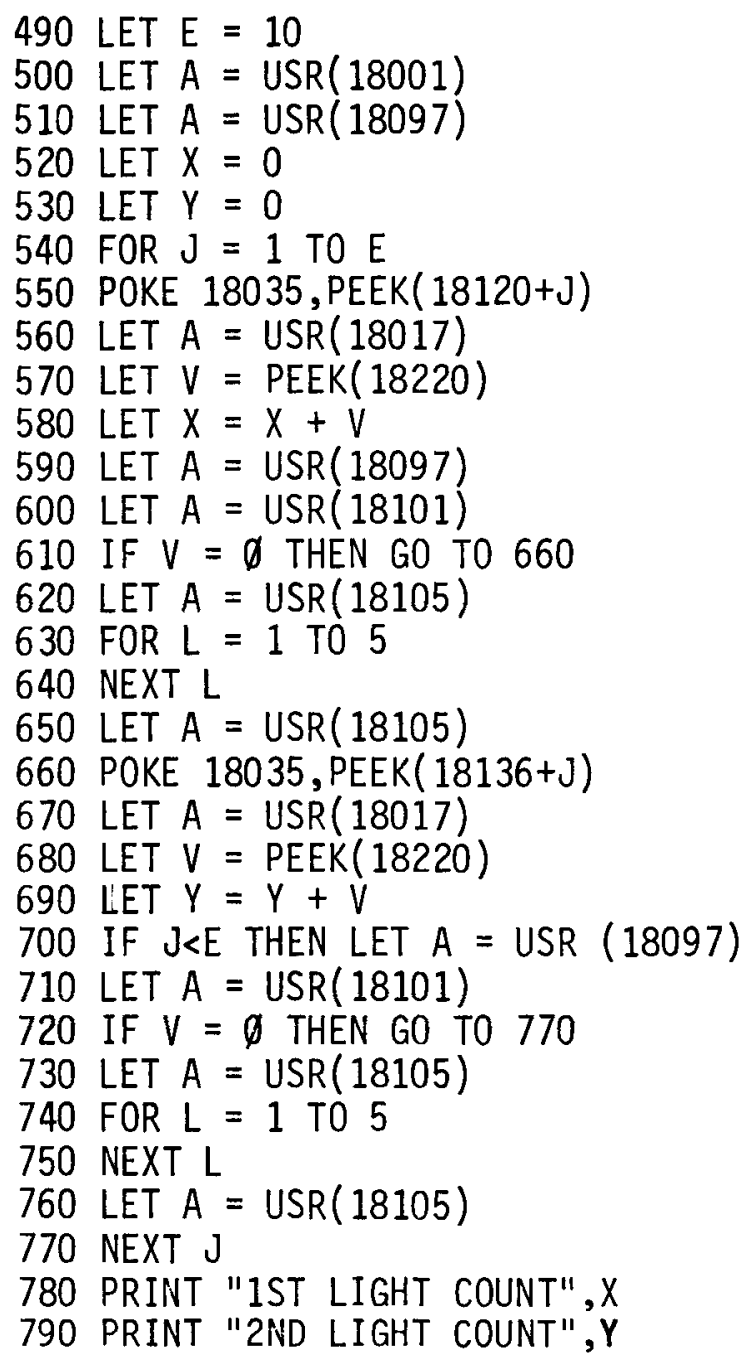

Figure 2. Program.

Only the count for the first position, B0, is used in the illustrative program. However, the routine beginning in Location 18017 has the potential, as it stands, for recording keypresses on eight lines. Indeed, the routine is making just such scans at all times. In line 570 , the number of leverpresses in the interval is retrieved from Memory Location 18220 and added to X in line 580 .

After the timing interval is over, Cue Light 1 is turned off in Statement 590, and Cue Light 2 is turned on in Statement 600 . In line 610 , the program sees whether a response has been made in the interval. If there has been one, the feeder is tumed on in line 620 , a delay is instituted in lines 630 to 640 , and the feeder is turned off again in line 650 . The feeder mechanism must be pulsed on, kept on for a short period, and pulsed off again.
The second half of the program, from lines 660 to 760 , is almost identical to the first half. Line 700 keeps Cue Light 1 turned off at the end if the program is completed. Lines 780 and 790 print out the results.

To summarize, a call through USR to Location 18001 initializes the program, one to 18097 changes the state of Cue Light 1, a call to 18101 changes the state of Cue Light 2, and a call to 18105 changes the state of the feeder mechanism. The results of the count during the time period are found in Locations 18220 and above. The time of the interval is specified in Location 18035. The times of the variable-interval schedules are found in remark lines 160 through 190 (in hexadecimal). Timing and counting an interval is begun by a call through USR to Location 18017.

Once these machine language constants are set up, 
programs of great complexity may be constructed, using only BASIC commands.

\section{DISCUSSION}

This $\$ 200$ computer controller is fully capable of running all aspects of an operant chamber. Further, with very little extra cost in additional electronic components, the system can run up to six operant chambers at one time. The eight input ports are already being scanned by the routine located in 18017 and beyond. Output lines A3 through A7 can be used to control feeder mechanisms in the other five boxes. The cue lights can be tied together. Of course, the BASIC program has to be changed somewhat, but the changes are not major; essentially, loops replace single statements.

The MicroAce has deficiencies. The keyboard is poor and the screen darkens when the machine is computing. The $2 \mathrm{~K}$ memory is not extensive, although I have not yet run out of memory. Input/output can be difficult (e.g., in the system described, one can read from Port B but not from Port A, because of hardware limitations). Finally, the machine is physically very unattractive, an important factor in some cases.

\section{REFERENCES}

Nichols, J. C., Nichols, E. A., \& RoNy, P. R. Z-80 microprocessor programming and interfacing: Book 2. Indianapolis, Ind: Sams, 1979.

REED, A. On choosing an inexpensive microcomputer for the experimental psychology laboratory. Behavior Research Methods \& Instrumentation, 1980, 12, 607-613.

(Received for publication August 1, 1981; revision accepted August 24, 1981.) 